\title{
Fertile offspring derived from mammalian eggs lacking either animal or
}

\section{vegetal poles}

\author{
Magdalena Zernicka-Goetz \\ Wellcome/CRC Institute, Tennis Court Road, Cambridge, CB2 1QR, UK \\ (e-mail: mzg@mole.bio.cam.ac.uk)
}

Accepted 29 September; published on WWW 9 November 1998

\section{SUMMARY}

In all animals so far tested, removing either pole of the undivided egg prevents normal development: embryos may arrest early, lack organs, or the adults may be sterile. These experiments have shown that spatial patterning of the egg is of utmost importance for subsequent development. However, the significance of spatial patterning in mammalian eggs is still controversial. To test the importance of egg polarity in the mouse a substantial amount of material either from the animal (polar bodyassociated) or the vegetal (opposite) pole of the fertilised egg was removed. One pole of the egg was cut away manually with a glass needle and the eggs were allowed to develop in vitro. Both kinds of surgical operation permit the development of blastocysts, which, after transfer to the uteri of pseudo-pregnant foster mothers, can produce viable offspring. Furthermore, these develop into fertile adult mice. I conclude that mouse eggs have no essential components that are localised uniquely to the animal or the vegetal pole and, therefore, do not rely for their axial development on maternal determinants that are so localised in the fertilised egg. Thus the mammalian egg appears to be very unusual in the animal kingdom in that it establishes the embryonic axes after the zygote has begun development.

Key words: Mammalian egg, Animal-vegetal polarity, Mouse

\section{INTRODUCTION}

Whether polarity of the mammalian egg - that is, its specific pattern of spatial organisation - is important in determining any aspect of further development has been a matter of continuing speculation and debate. A number of experiments have shown that the early mouse embryo has considerable capacity to regulate its development (reviewed by Rossant and Pedersen, 1986). Cleavage stage embryos can be aggregated to form chimeras, and individual blastomeres can be removed or displaced, and yet development can proceed normally. For example, Kelly $(1975,1977)$ demonstrated that all blastomeres at the 4-cell stage and some at the 8-cell stage retained the ability to form both trophectoderm and inner cell mass. Together these experiments would argue that the specific developmental fate of cells has not been determined at these stages, and imply that important maternal factors are not localised in the egg. However, the interpretation of many of these experiments has been questioned (reviewed by Gardner, 1996). In the case of Kelly's work, for example, technical difficulties made it impossible to establish whether all blastomeres at the 8-cell stage remain totipotent. This is a particularly important question as once the third cleavage has occurred, cytoplasm from different axial levels of the egg is partitioned into individual blastomeres.

The conclusion that the polarity of the mammalian egg is developmentally insignificant has been found surprising and therefore questioned because in all other animals tested, such as ascidians (Bates and Jeffery, 1987; Jeffery, 1990), flies (St Johnston and Nüsslein-Volhard, 1992), sea urchins (Horstadius, 1973; Horstadius, 1973; Logan and McClay, 1997) and frogs (Gurdon et al., 1985) determinants for normal development are already localised in the 1-cell egg. In the sea urchin, for example, the importance of animal-vegetal polarisation is evident when the egg is bisected into animal or vegetal parts. Whereas a vegetal half of the egg is able to develop to give an almost normal larva, an animal half becomes an abnormal blastula (Horstadius, 1973). Furthermore, removal of as little as $8 \%$ of the total egg volume of vegetal pole cytoplasm from the starfish egg prevents archenteron development (Kuraishi and Osanai, 1994). Similarly, if a small region (5-8\%) of the egg cytoplasm is removed from the vegetal pole of the ascidian egg, most embryos will fail to gastrulate (Nishida, 1996). Thus, in these and in other species that have been studied, the vegetal pole of the egg is crucial for spatial determination in later development.

New results have re-awakened interest in the significance of egg polarity for mammalian development. Most importantly, studies by Gardner (Gardner, 1997) indicate that the mouse blastocyst has an axis of bilateral symmetry that relates to the polarity of the egg as defined by the position of the polar body. In addition, Antczak and van Blerkom (1997) have shown that there is an asymmetric distribution of the cytokine leptin and the signalling molecule STAT3 to the animal pole of the mouse and human egg. As a consequence of the orientation of the cleavage planes, these proteins become allocated preferentially 
to specific blastomeres and subsequently to the cells of the trophectoderm rather than cells of the inner cell mass. Collectively these observations have led to the hypothesis (Antczak and Van Blerkom, 1997; Edwards and Beard, 1997) that egg polarity determines the spatial patterning of the blastocyst and succeeding stages of mammalian development. However, this hypothesis has not been tested experimentally. To determine the significance of egg polarity on the establishment of body axes in mammals, either the animal or the vegetal pole was removed from mouse eggs and the effects of this operation on subsequent development was examined.

\section{MATERIALS AND METHODS}

Eggs used to perform these experiments were collected after spontaneous ovulation from $\mathrm{F}_{1}$ (C57BL/6xCBA) females mated with males of the same genotype. The eggs were released from the ampullae into flushing and handling Medium 2 (M2) containing $4 \mathrm{mg} / \mathrm{ml}$ of bovine serum albumin (M2 with BSA) and containing hyaluronidase (200 i.u./ml) to remove the cumulus cells. The zona pellucida was then removed with the help of brief exposure to acid Tyrode's solution as previously described (Zernicka-Goetz et al., 1995). Zona-free eggs were always left for at least 30 minutes in KSOM (Speciality media, Inc. Lavallette, $\mathrm{NJ}$ ) with $4 \mathrm{mg} / \mathrm{ml}$ of BSA before microsurgery.

Eggs were cut by hand under a dissecting microscope with a glass needle whose diameter was about half the diameter of the egg. Cutting

A
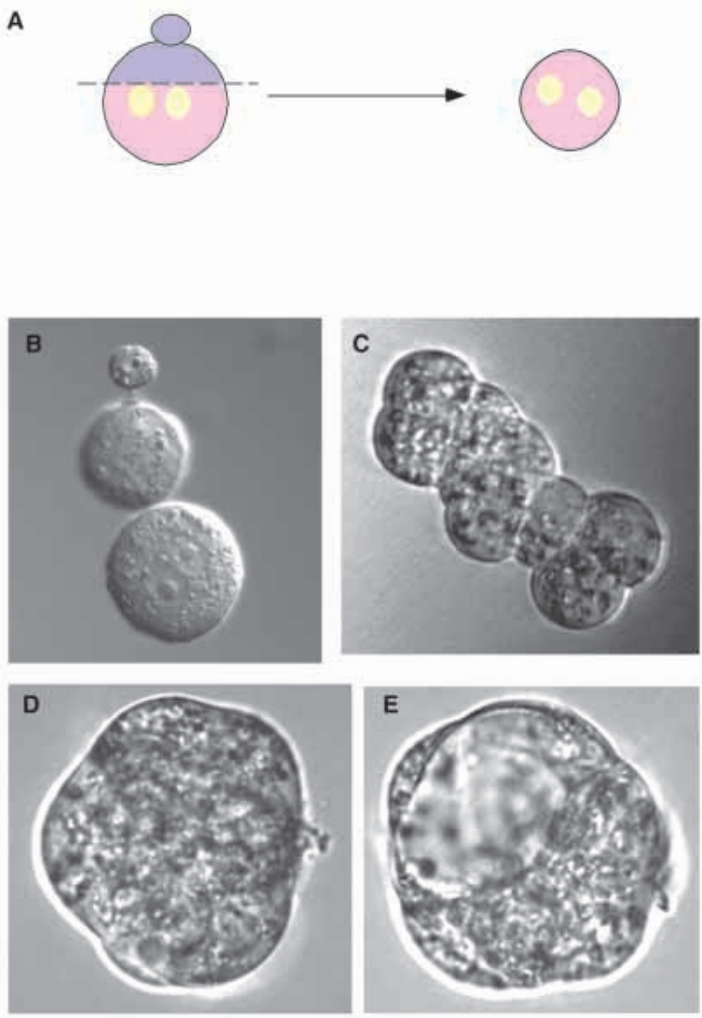

Fig. 1. Development of an egg lacking the animal pole. (A) Scheme to illustrate the method of bisection. (B) Transmitted light image of a representative egg immediately after cutting to remove the animal fragment. The animal fragment (the upper part) with its attached polar body was discarded, and the vegetal fragment (the lower part) was cultured. The diameter of an oocyte is approximately $80 \mu \mathrm{m}$. (CE) Subsequent development of the vegetal fragment to the 8-16-cell stage (C), morula stage (D) and blastocyst (E). was accomplished about 3-5 hours before first mitotic division of the zygote. The operation was performed in M2 containing $4 \mathrm{mg} / \mathrm{ml} \mathrm{BSA}$ at room temperature on a surface of $1 \%$ agar dissolved in $0.6 \% \mathrm{NaCl}$. The agar plate and the cutting medium were cooled before the operation to $5^{\circ} \mathrm{C}$. The eggs were cut in groups of 10 and then transferred into the culture medium. The survival rate varied between experiments. In some experiments the majority of eggs survived, whereas in other cases as many as $50 \%$ of the eggs lysed. Eggs that were damaged during bisection lysed immediately after cutting and were discarded. Embryos were cultured up to the blastocyst stage, in KSOM supplemented with amino acids (Speciality media, Inc. Lavallette, NJ) and BSA (4 mg/ml) in an atmosphere of $5 \% \mathrm{CO}_{2}$ at $37^{\circ} \mathrm{C}$.

Eggs were cut in relation to the position of the polar body. The location of the polar body defines the animal pole of the egg, and the diametrically opposite point on its surface is termed the vegetal pole (Balinsky, 1970). During the cutting operation the polar body remained attached to the egg and therefore provided a reliable marker of the animal pole. Cutting was accomplished as described by Tarkowski (Tarkowski and Rossant, 1976) except that the needle was applied to the egg equatorially and then the whole egg was pressed gently. The needle was subsequently slowly moved in the direction of one of the poles, and finally pressed firmly to cut away a portion of the egg. As a control, eggs were cut meridionally. Often the fragments remained connected by a cytoplasmic bridge. The two fragments could then be separated by cutting the bridge with a fine needle. Eggs and embryos were photographed using a cooled CCD camera
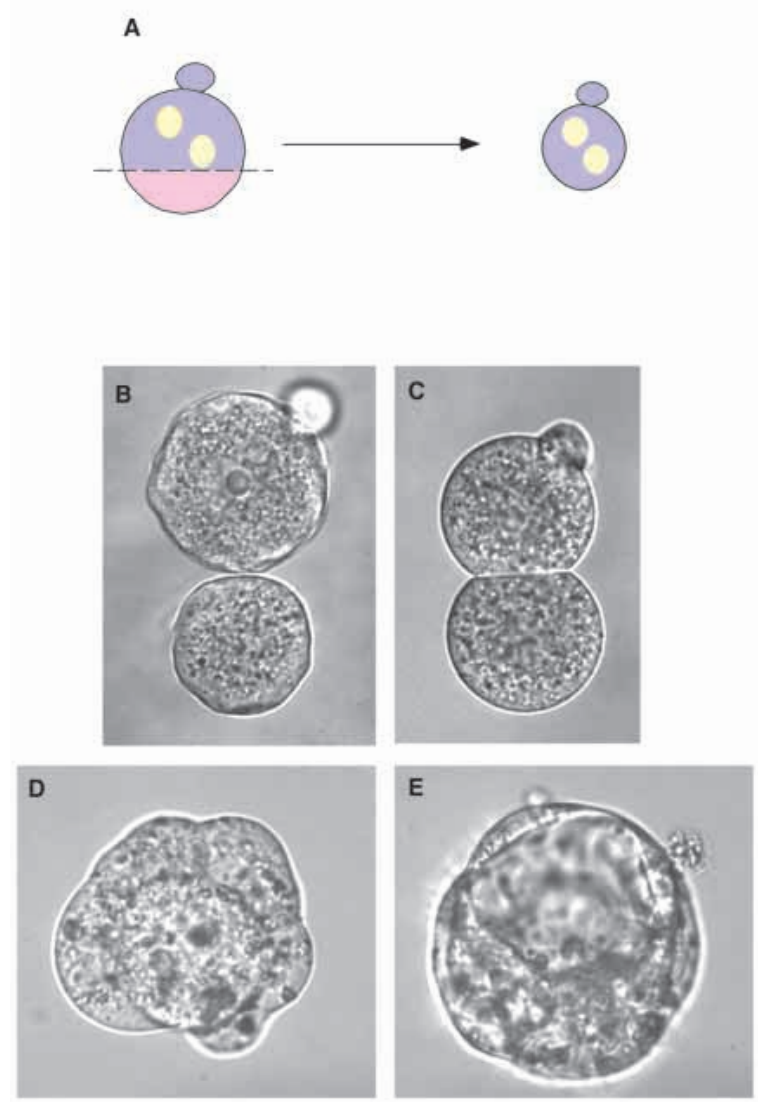

Fig. 2. Development of an egg lacking the vegetal pole. (A) Scheme to illustrate the bisection. (B) Transmitted light image of a representative egg immediately after cutting to remove the vegetal pole. The animal fragment (the upper part) with its attached polar body was cultured, and the vegetal fragment (the lower part) was discarded. (C-E) Subsequent development of the animal fragment to the 2 cell stage (C), morula stage (D), and blastocyst (E). 
(Princeton Instruments, USA) and DIC optics on a Nikon inverted microscope. The percentage of cytoplasmic volume which was removed by this procedure was calculated from the diameter of the animal or vegetal fragments of the egg using IP Lab Spectrum image processing software (Scanalytics, USA). The proportion of cytoplasm removed when eggs were cut so as to discard animal, vegetal or longitudinal fragments was between 12 to $29 \%$ (average 20\%), 20 to $52 \%$ (average $34 \%$ ), and 12 to $42 \%$ (average $25 \%$ ) respectively. Egg fragments were transferred separately to drops of culture medium under mineral oil in plastic dishes and cultured for a further 4 days in vitro. Chimaeric embryos were formed by aggregating two 2-cell stage embryos derived from eggs lacking a specific pole.

Late morulae or blastocysts derived from eggs lacking the animal or vegetal pole were transferred into the uteri of pseudo-pregnant mothers of the albino MF1 strain that had been mated 2.5 days earlier with vasectomised males. Great care was taken to select blastocysts derived from eggs from which not less than approximately $20 \%$ of the cytoplasm was removed. The experimental embryos were transferred together with 'carrier' blastocysts of MF1 strain of mice to control for the success of transfer. Such carrier embryos were contained within a zona pellucida, and had not been subjected to any in vitro culture.

\section{RESULTS}

In order to determine whether there is a requirement for polar cytoplasm for the early development of the mouse embryo,
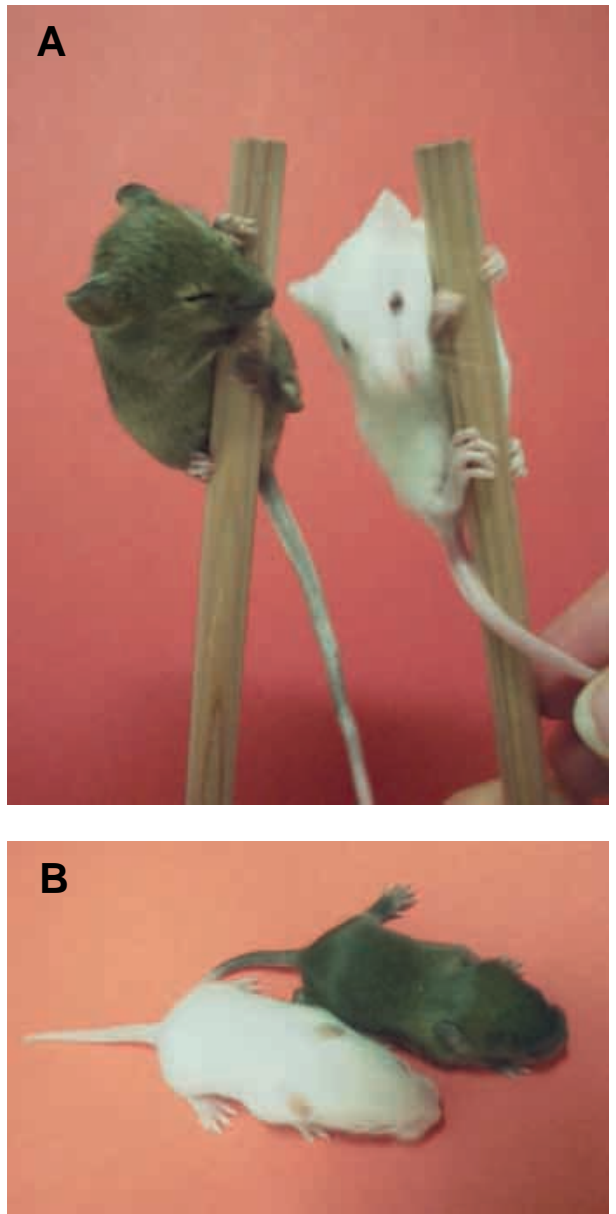

Fig. 3. New-born mice derived from eggs lacking an animal or a vegetal pole. (A) Agouti mouse born from an egg without a vegetal pole or (B) without an animal pole with their albino foster brothers born from carrier embryos. either pole of the egg was cut away with a glass needle and the egg was allowed to develop in vitro. The animal-vegetal (AV) axis was defined by the position of the second polar body, and the plane of cutting was related to this axis. In the majority of eggs, the pronuclei were visible at the time of microsurgery, which was carried out in such a way that one of the resulting fragments of the embryo retained both the maternal and paternal pronuclei.

To assess the importance of any potential animal pole determinants, eggs were bisected perpendicularly to the AV axis to remove as much of the cytoplasm as possible from the animal polar region (Fig. 1A,B). To ensure that the residual portion of the egg remained diploid, the eggs were cut after the female pronucleus had begun to move to the centre of the egg. This bisection removed, on average, $20 \%$ of the total egg volume from the animal part of the egg (see Materials and Methods). Eggs lacking the animal pole often developed into elongated embryos at the 8-16 cell stage (Fig. 1C), a morphology that also arose in some control embryos cultured without a zona. Nevertheless, $80 \%$ (49/61, Table 1) of the experimental embryos compacted and developed into spherical morulae (Fig. 1D), and subsequently into apparently normal, although small, blastocysts (Fig. 1E). As a control, eggs were bisected parallel to the $\mathrm{AV}$ axis so as to leave both pronuclei
A
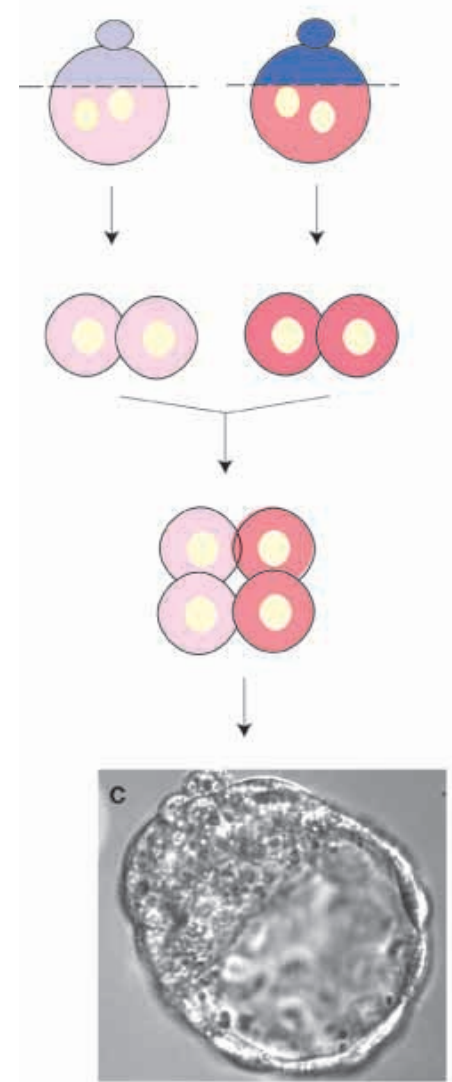

B
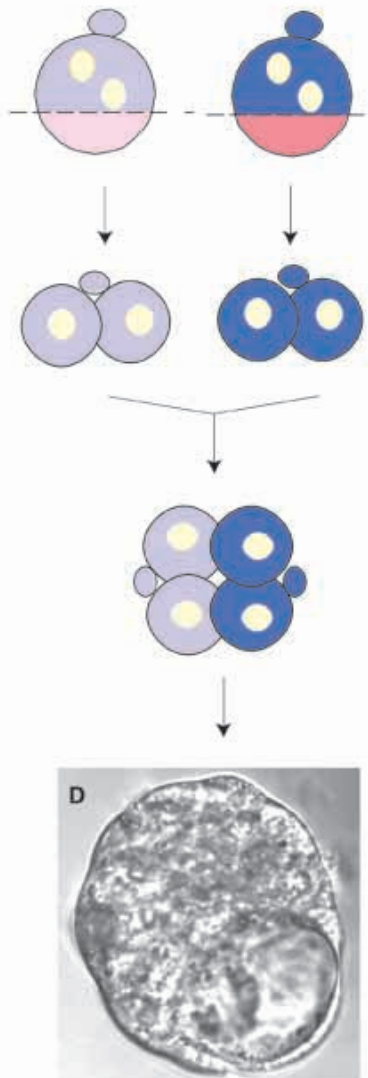

Fig. 4. Development of chimaeric embryos derived from eggs without one or the other pole. (A,B) Schemes to illustrate how chimaeras were formed from eggs without the animal pole (A) or without the vegetal pole (B). (C,D) Representative blastocyst of chimaeras derived from eggs without either the animal pole (C) or the vegetal pole (D). 
Table 1. Developmental potential of eggs lacking either an animal or a vegetal pole

\begin{tabular}{|c|c|c|c|c|c|c|}
\hline \multirow[b]{2}{*}{ Treatment of egg } & \multirow[b]{2}{*}{ Total number } & \multirow{2}{*}{$\begin{array}{l}\text { Number develop } \\
\text { to morula/blastocyst }\end{array}$} & \multirow[b]{2}{*}{ Number transferred } & \multirow[b]{2}{*}{ Live born } & \multicolumn{2}{|c|}{ Fertile adults } \\
\hline & & & & & Male & Female \\
\hline Animal pole cut out & 61 & 49 & 35 (+17 carriers $)$ & $2 *(+14$ carriers $)$ & - & 1 \\
\hline Vegetal pole cut out & 149 & 122 & 40 (+17 carriers $)$ & 2 (+11 carriers $)$ & 2 & - \\
\hline Meridional cut (control) & 78 & 60 & 18 (+7 carriers) & $2 \dagger(+2$ carriers $)$ & 1 & - \\
\hline Animal pole cut out chimaeras & 11 & 10 & 8 (no carriers) & $5 \S$ & 1 & 2 \\
\hline Vegetal pole cut out chimaeras & 29 & 27 & $14(+4$ carriers $)$ & $3(+1$ carrier $)$ & 3 & - \\
\hline
\end{tabular}

in the same fragment of the egg, removing on average $25 \%$ of the cytoplasm. A similar fraction (77\%, 60/78, Table 1) of these control embryos developed to the blastocyst stage.

To assess the importance of the vegetal pole for embryonic development, an average of $34 \%$ of the total volume of the egg cytoplasm was cut away, as shown in Fig. 2A,B. Interestingly, this manipulation resulted in a change in the cleavage plane of the first division in more than half of the eggs (Fig. 2C). Instead of cleaving normally (meridionally), isolated animal halves had some preference $(56 \%, 83 / 149)$ to divide perpendicularly to the AV axis. This alteration in cleavage plane had no apparent effect on their developmental potential, because embryos developed to the blastocyst stage with the same success, regardless of the plane of the first division. A large majority $(82 \%, 122 / 149$, Table 1$)$ of eggs cut to remove vegetal pole cytoplasm developed in an apparently normal fashion to the morula stage (Fig. 2D), and subsequently to the blastocyst (Fig. 2E).

In order to determine whether the change in the orientation of the first cleavage plane resulted from the loss of vegetal cytoplasm, the eggs were separated into animal and vegetal fragments, and allowed to fuse immediately after cutting. In this case there was some change in the orientation of the cleavage, but it was reduced to $36 \%$ (39/94) of embryos as compared to $56 \%(83 / 149)$ in embryos that had been completely bisected perpendicular to the AV axis. When eggs were cut along the AV axis, 29\% (26/90) of them changed the plane of first cleavage. Because these fragments contained both animal and vegetal cytoplasm, the alteration in cleavage plane could be attributed to perturbing the cellular architecture of the egg rather than to changing its composition. Moreover, because cutting eggs perpendicularly to the $\mathrm{AV}$ axis caused more of them (56\%) to change the cleavage plane than cutting meridionally (29\%), there may be components that determine the orientation of cleavage distributed along the equatorial region of the egg.

The definitive test of an egg's developmental potential would be its capacity to complete embryogenesis to term. Therefore, blastocysts derived from eggs that were lacking an average of $20 \%$, or of $34 \%$, of cytoplasm from their animal or vegetal poles respectively, were transferred to the uteri of pseudopregnant mothers, together with genetically distinct carrier embryos that had neither been manipulated nor cultured in vitro. In these experiments, two embryos derived from eggs lacking the animal pole, and two from those lacking the vegetal pole, developed normally to term (Fig. 3A,B). This corresponds to $5 \%(2 / 40)$ to $6 \%(2 / 35)$ of the bisected embryos that were transferred to recipient uteri (Table 1). A similar proportion $(11 \%, 2 / 18)$ of embryos developed to term from blastocysts derived from eggs cut meridionally and which therefore retained both animal and vegetal cytoplasm. In these experiments $62 \%$ (28/45) of carrier MF1 blastocysts developed into new-born mice.

It was possible that the low developmental potential was a consequence of the reduction in the number of cells in the blastocysts derived from experimentally manipulated eggs. To test this, the number of cells in the embryos was increased by aggregating two embryos derived from eggs lacking a specific pole (Fig. 4). This substantially increased the proportion of embryos that developed to term. $62 \%(5 / 8)$ of chimaeric blastocysts derived from two embryos lacking animal poles, and $21 \%(3 / 14)$ of chimaeric blastocysts derived from two embryos lacking vegetal poles, developed into new-born mice (Table 1).

It was conceivable that the mice that developed from the manipulated eggs might be sterile if there were determinants at the poles that were required for the proper development of the germ line. However, the animals reported here proved to be fertile. Specifically, two agouti male mice that developed from eggs lacking a vegetal pole produced normal litters of healthy pigmented new-born mice when mated with albino MF1 females (11 in one case and 14 in the second). Similarly, an agouti female derived from an egg lacking an animal pole gave birth to 7 normal offspring when mated with an albino male.

\section{DISCUSSION}

Recent studies suggesting a relationship between the polarity of the egg and the development of symmetry in the preimplantation embryo have led to the hypothesis that egg polarity is functionally important in axis formation in the mammalian embryo, as it is in other animals (Antczak and van Blerkom, 1997; Edwards and Beard, 1997). This study was designed to test this hypothesis by removing a substantial amount of material, either from the animal or the vegetal pole of the fertilised egg. Eggs subjected to either surgical operation were able to develop into blastocysts. Therefore, there appear to be no essential determinants for development to the blastocyst stage that are localised uniquely to either the animal or the vegetal pole of the fertilised mouse egg.

It is intriguing that in mammals the orientation of the patterns of the cleavage divisions is usually very regular. In fact, Gardner (1997) has observed that during the course of unperturbed development, the pattern in which the egg cleaves results in the preferential localisation of the polar body to a 
specific position at the blastocyst stage. However, my results show that successful development does not depend on whether an embryo first cleaves equatorially or meridionally. During normal development the first division is in almost all cases parallel to the AV axis of the egg. Consequently, material from both animal and vegetal poles is present in both blastomeres at the 2-cell stage. After cutting eggs orthogonally to the AV axis, I observed that instead of cleaving normally, isolated animal halves had a tendency to divide perpendicularly to the AV axis. However, the embryos developed into blastocysts with the same success regardless of the plane of the first division. Thus, it would seem that the sequence in which cytoplasmic components of the egg are distributed into specific blastomeres has little or no consequences for subsequent development.

The definitive demonstration of the developmental potential of preimplantation embryos developing from eggs without one or the other pole, is their capacity to complete embryogenesis. The two mice born from eggs lacking the animal pole, and the two from those lacking the vegetal pole correspond to $5 \%$ $(2 / 40)$ to $6 \%(2 / 35)$ of the number of bisected embryos transferred to recipient uteri. Although the number of experimental embryos surviving to term appears small, a similar proportion $(11 \%, 2 / 18)$ of blastocysts derived from eggs cut meridionally successfully developed to term. Moreover, a similar percent ( 8 to $12 \%$ ) of embryos developed to term after a much less radical manipulation in the production of monozygotic twins: 8- to 16-cell stage embryos were bisected and transferred to recipient uteri (Tsunoda and McLaren, 1983).

The low proportion of embryos developing to term can be explained by a number of factors. Eggs without an animal pole or without a vegetal pole, or cut meridionally, developed more slowly than uncut embryos, and the resulting blastocysts were smaller and had fewer cells than normal. A similar situation was described when eggs were cut to produce haploid halves (Tarkowski, 1977; Tarkowski and Rossant, 1976). The size of the blastocysts that developed from animal and vegetal fragments of the eggs varied inversely with the proportion of egg cytoplasm that had been cut away. Therefore, the reduced number of cells in the blastocyst may be a consequence of the change in the nucleo-cytoplasmic ratio, as has been previously suggested (Tarkowski, 1977). Blastocysts with a reduced number of inner cell mass cells have a severely compromised developmental potential (Ansell and Snow, 1975). In agreement with this, when I increased the number of cells by aggregating two 2-cell stage embryos derived from eggs lacking one or the opposite pole the resulting blastocysts had a greatly increased chance of survival. Doubling the number of cells increased the proportion of embryos that developed to term by four to ten fold. The viability of all the experimental embryos could also have been influenced by other factors such as operating on eggs at the 1 cell stage and culturing them without the zona pellucida in vitro for 4 days before their transfer to foster mothers. Thus, it is all the more significant that the manipulated eggs in this study developed to the blastocyst stage and beyond.

Not only could animals develop from eggs lacking the animal or vegetal poles, but these mice developed into fertile adults. Thus it would also appear that the mouse egg does not have determinants at the poles that are required for the proper development of the germ line. In this respect the mouse egg also differs significantly from those of certain invertebrates, as well as amphibian embryos (Houston et al., 1998; Lehmann and Ephrussi, 1994; Zhou and King, 1996) where cytoplasmic determinants for germ line development are localised maternally to polar regions.

In conclusion, the mice born in the experiments presented in this paper are the first mammals reported to develop from eggs lacking vegetal or animal poles. Thus, they provide compelling evidence that there are no egg determinants that are localised uniquely to either animal or vegetal pole which are essential for any aspect of the further development of the mammalian egg. However, because the definitive embryo does have polarity it is likely that there are specific components whose asymmetry is established, or can be re-established, later in development. Even if so, the mammalian egg appears to be very unusual in the animal kingdom.

I thank my colleagues John Gurdon, Jonathon Pines, Roger Pedersen, Christopher Graham, Anne McLaren and Azim Surani for their encouragement and useful comments during the course of this work. I am grateful to Martin Evans for his continuing support, including providing space. I thank David Glover for discussions and for help in photographing mice. I also thank Richard Gardner for drawing my attention to the unresolved issue of the role of polarity in the mammalian egg. I thank the Lister Institute of Preventive Medicine. This work was also supported by Wellcome Trust grants to Martin Evans and to M. Z. G.

\section{REFERENCES}

Ansell, J. and Snow, M. (1975). The development of trophoblast in vitro from blastocysts containing varying amounts of inner cell mass. J. Embryol. exp. Morph. 33, 177-185.

Antczak, M. and Van Blerkom, J. (1997). Oocyte influences on early development: the regulatory proteins leptin and STAT3 are polarized in mouse and human oocytes and differentially distributed within the cells of the preimplantation stage embryo. Mol. Hum. Reprod. 3, 1067-1086.

Balinsky, B. I. (1970). An Introduction to Embryology. 3rd edn. Philadelphia: W. B. Saunders Company.

Bates, W. and Jeffery, W. (1987). Localization of axial determinants in the vegetal pole region of ascidian eggs. Dev. Biol. 1245, 66-76.

Edwards, R. G. and Beard, H. K. (1997). Oocyte polarity and cell determination in early mammalian embryos. Mol. Hum. Reprod. 3, $863-$ 905

Gardner, R. (1996). Can developmentally significant spatial patterning of the egg be discounted in mammals? Hum. Repr. 2, 3-27.

Gardner, R. (1997). The early blastocyst is billaterally symmetrical and its axis of symmetry is aligned with the animal-vegetal axis of the zygote in the mouse. Development, 124, 289-301.

Gurdon, J. B., Mohun, T. J., Fairman, S. and Brennan, S. (1985). All components required for the eventual activation of muscle-specific actin genes are localized in the subequatorial region of an uncleaved amphibian egg. Proc-Natl. Acad. Sci. USA 82, 139-143.

Horstadius, S. (1973). Experimental Embryology of Echinoderms. Oxford: Clarendon Press.

Houston, D. W., Zhang, J., Maines, J. Z., Wasserman, S. A. and King, M. L. (1998). A Xenopus DAZ-like gene encodes an RNA component of germ plasm and is a functional homologue of Drosophila boule. Development 125, 171-180.

Jeffery, W. (1990). Ultraviolet irradiation during ooplasmic segregation prevents gastrulation, sensory cell induction, and axis formation in the Ascidian embryo. Dev. Biol. 140, 388-400.

Kelly, S. J. (1975). Studies of the potency of early cleavage blastomeres of the mouse. In The Early Development of Mammal.s (ed. Balls, M. and Wild, A. E.), pp. 97-105. Cambridge: Cambridge University Press.

Kelly, S. J. (1977). Studies of the developmental potential of 4- and 8-cell stage mouse blastomeres. J. Exp. Zool. 200, 365-376.

Kuraishi, R. and Osanai, K. (1994). Contribution of maternal factors and 
cellular interactions to determination of archenteron in the starfish embryo. Development, 120, 2619-2628.

Lehmann, R. and Ephrussi, A. (1994). Germ plasm formation and germ cell determination in Drosophila. Ciba Found. Symp. 182, 282-296.

Logan, C. Y. and McClay, D. R. (1997). The allocation of early blastomeres to the ectoderm and endoderm is variable in the sea urchin embryo. Development 124, 2213-2223.

Nishida, H. (1996). Vegetal egg cytoplasm promotes gastrulation and is responsible for specification of vegetal blastomeres in embryos of the ascidian Halocynthia roretzi. Development 122, 1271-1279.

Rossant, J. and Pedersen, R. (1986) Experimental Approaches to Mammalian Embryonic Development Cambridge: Cambridge University Press.

St Johnston, D. and Nüsslein-Volhard, C. (1992). The origin of pattern and polarity in the Drosophila embryo. Cell 68, 201-219.

Tarkowski, A. (1977). In vitro development of haploid mouse embryos produced by bisection of one cell fertilized eggs. J. Embyol. Exp. Morph. 38, 187-202.

Tarkowski, A. and Rossant, J. (1976). Haploid mouse blastocysts developed from bisected zygotes. Nature 259, 663-665.

Tsunoda, Y. and McLaren, A. (1983). Effect of various procedures on the viability of mouse embryos containing half the normal number of blastomeres. J. Reprod. Fert. 69, 315-322.

Zernicka-Goetz, M., Ciemerych, A. M., Kubiak, J. Z., Tarkowski, A. K. and Maro, B. (1995). Cytostatic factor inactivation is induced by a calcium-dependent mechanism present until the second cell cycle in fertilised but not in parthenogenetically activated mouse eggs. J. Cell Sci. 108, 469-474.

Zhou, Y. and King, M. L. (1996). Localization of Xcat-2 RNA, a putative germ plasm component, to the mitochondrial cloud in Xenopus stage I oocytes. Development 122, 2947-53. 